intensity with wave-length, with power and with aerial directivity all seem to confirm this, for these are the factors which determine the intensity of a received scatter signal itself.

It is generally assumed that ionosphere storms of the kind mentioned are caused by corpuscles ejected from the sun, the effect of tioned are caused by corpuscles ejected from the sun, the effect of even negligible at the height of the $E$ laver. If the 'rumble' is indeed oven negligible at the height of the $E$ laver. If the rumble' is indeed do produce a marked effect upon the $E$ layer, at least upon the ionic do produce a marked effect upon the $E$ layer, at least upon the ionic clouds, if not upon the regular structure of the ionization. As to explanation, but it is suggested that the clouds are affected in such a way that a kind of Doppler effect is imparted to the scattered energy. way that a kind of Doppler effect is imparted to the scattered energy. A further point is that the above postulation as to the cause of the 'rumble' would provide additional confirmation of the evidence of Bckersley, Millington and Cox that the scatter is indeed from a poir
in the atmosphere and not from a distant point on the ground.

Engineering Division,

B.B.C., London, W.1. Feb. 18.

${ }^{1}$ Eckersley, T. L., Nature, 143, 33 (1939).

Eckersley, T. L., J. Inst. Elect. Eng. 86, 658 (1940).

Eckersley, T. L., Millington, G., and Cox, J. W., Nature, 153, 341 (1944).

\section{Shape of Collision-Broadened Spectral Lines}

Van Vleck and Weisskopf ${ }^{1}$, in an interesting paper, have shown that the well-known Lorentz formula for the shape of a spectral line is incorrect except at frequencies close to the resonance frequency. Since this formula has been considered to be correct for a great numbe of years, it may be of interest to record that, using a different method I had reached the same conclusions ${ }^{2}$ as Van Vleck and Weisskopf This method, sithough originally developed for the case of a rigid dipole oscillating about an equilibrium position, can easily be adapted to harmonic oscillators.

Consider an assembly of $N$ equal harmonic oscillators of which the proper frequency, mass, charge and displacement are denoted by $\omega_{0} / 2 \pi$, $M, e, x$, respectively. In an external feld $F, x$ satisfles

$$
\ddot{x}=-\omega_{0}{ }^{2} x+e F / M, .
$$

whereas the energy is given by

$E=E_{0}-e F x$, where $E_{0}=\frac{M}{2} \dot{x}^{2}+\frac{M}{2} \omega_{0}^{2} x^{2}$.

In a constant field $F=F_{0}$, the distribution function $f(x, \dot{x})$ (that is number of oscillators per unit range of $x$ and $\dot{x}$ ) is proportional to exp. $-E_{0} / k T$. For sufficiently weak flelds, $f=f_{0}+f_{1}$, where

$$
f_{0}=C \exp \cdot\left(-E_{0} / k T\right), \quad \cdots \int_{-\infty}^{\infty} f_{0} d x d \dot{x}=N,
$$

and

$$
f_{1}=C \exp .\left(-E_{0} / k T\right) \frac{e F_{0} x}{k T}=-\frac{e F_{0}}{M \omega_{0}^{2}} \frac{\partial f_{0}}{\partial x},\left|f_{1}\right| \ll f_{0} .
$$

The polarization is thus given by

$$
\begin{aligned}
& P_{\theta}=e \int x f d x d \dot{x}=e \int x f_{1} d x d \dot{x}= \\
& -\frac{e^{2} F_{0}}{M \omega_{0}{ }^{2}} \int x \frac{\partial f_{0}}{\partial x} d x d \dot{x}=\frac{e^{2} N F_{0}}{M \omega_{0}{ }^{2}} .
\end{aligned}
$$

Consider now a periodic fleld $F^{\prime} \Rightarrow F_{0}$ exp. $i \omega t$. If instantaneous equilibrium could be reached, the distribution function would be

$$
f_{\text {equ. }}=f_{0}+f_{1} \exp . i \omega t \text {. }
$$

Assume the actual distribution function to be

$$
f=f_{0}+f_{2} \exp . i \omega t, \ldots\left|f_{2}\right| \ll f_{0} .
$$

At any instant of time the oscillators will then tend to reach $f$ equ if they are in interaction (collisions) with a medium which is in thermal equilibrium. The main assumption of the present calculation is to equilibrium. The main assumption of the present calculation is to suppose that the rate of change of $f$ due to these collisions is given
by $-(f-f$ equ. $) / \tau$, where the time of relaxation $\boldsymbol{r}$ may depend on temperature but is independent of $x$ and $\dot{x}$. Adding the rate of change due to oscillation, we find

$$
\frac{\partial f}{\partial t}=\frac{\partial f}{\partial x} \dot{x}+\frac{\partial f}{\partial \dot{x}} \ddot{x}-\frac{1}{\tau}\left(f-f_{\text {equ. }}\right)
$$

or making use of (1), (5) and (6)

$$
\begin{array}{r}
\frac{\partial f}{\partial t}=\frac{\partial f}{\partial x} \dot{x}-\omega_{0}{ }^{2} \frac{\partial f}{\partial \dot{x}} x+\frac{e F_{0}}{M} \frac{\partial f}{\partial \dot{x}} \exp . i \omega t- \\
\frac{1}{\tau}\left(f_{2}-f_{1}\right) \text { exp. i } \omega t . . .
\end{array}
$$

Now insert (6) and (3) in (8), neglect terms in $F, f$, which are small of the second order, and notice that $\partial f_{0} / \partial t=0$. Then

$$
\begin{aligned}
\left(i \omega+\frac{1}{\tau}\right) f_{2}= & \frac{\partial f_{2}}{\partial x} x-\frac{\partial f_{2}}{\partial \dot{x}} \omega_{0}^{2} x+ \\
& \frac{e F_{0}}{M} \frac{\partial f_{0}}{\partial \dot{x}}-\frac{e F_{0}}{\tau M \omega_{0}^{2}} \frac{\partial f_{0}}{\partial x} . \quad . \quad . \quad .
\end{aligned}
$$

This equation can be solved by putting

$$
f_{2}=a \frac{\partial f_{0}}{\partial x}+b \frac{\partial f_{0}}{\partial \dot{x}}, \quad . \quad . \quad \text {. }
$$

where

$$
\begin{aligned}
a=-\frac{e F_{0}}{M \omega_{0}^{2}} \cdot \frac{1}{2} & \left\{\frac{1+i \omega_{0} \tau}{1+i\left(\omega+\omega_{0}\right) \tau}+\frac{1-i \omega_{0} \tau}{1+i\left(\omega-\omega_{0}\right) \tau}\right\}, \\
b & \left.=\frac{\tau}{1+i \omega \tau}\left(a \omega_{0}^{2}\right]+\frac{e F_{0}}{M}\right) . \text {. (11) }
\end{aligned}
$$

The total polarization $P$ is thus given by (if we note that $\int x \frac{\partial f_{0}}{\partial \dot{x}}=0$, and make.use of (11) and (4))

$$
\begin{array}{r}
P=e \int x f d x d \dot{x}=e \int x f_{2} \exp .(i \omega t) d x d \dot{x}=e a \exp . i \omega t \\
\int x \frac{\partial f_{0}}{\partial x} d x d \dot{x}=\frac{1}{2}\left\{\frac{1+i \omega_{0} \tau}{1+i\left(\omega+\omega_{0}\right) \tau}+\frac{1-i \omega_{0} \tau}{1+i\left(\omega-\omega_{0}\right) \tau}\right\} \\
P_{0} \exp . i \omega t, \quad \text {. . . (12) }
\end{array}
$$

in agreement with Van Vleck and Weisskopf's equations (17) (18), which are obtained as the real and imaginary parts (the latter multi. plled by $\omega$ ) of $P / F$.

$$
\begin{aligned}
& \text { H. H. Wills Physical Laboratory, } \\
& \text { University of Bristol. }
\end{aligned}
$$$$
\text { Jan. } 10 \text {. }
$$

\begin{tabular}{|c|c|}
\hline Ferrous oxide phase formed on: & Lattice parameter (A.) \\
\hline $\begin{array}{l}\text { Uncoated iron } \\
\text { MgO-coated iron }\end{array}$ & $\begin{array}{ll}\text { (a) } 4 \cdot 298^{4 \cdot 284} & \text { (b) } 4 \cdot 317\end{array}$ \\
\hline Pure MgO & $4 \cdot 230$ \\
\hline
\end{tabular}

'Van Vleck and Weisskopf, Rev. Mod. Phys., 17, 227 (1945). Addendum to Report L/T. 157 and Report L/T. 163 of the British Electrical and Allied Industries Research Association.

\section{'Impregnation' of Defect Lattices in Iron Oxide Scales}

IN the course of an X-ray examination on scales formed on steels. a structure phenomenon was observed which is believed to be of general interest. Magnesia is known to possess a considerably smaller lattice-parameter than ferrous oxide, with which it is isomorphous. If, therefore, wüstite (the $\mathrm{FeO}$ solid solution) dissolves magnesium oxide, its parameter would be expected to decrease, as the smaller Ma was allowed to form, by coating Armco iron with pure magnesla, and oxidizing in air at $1,000^{\circ} \mathrm{C}$. , the scale was then examined by powder-photographs. The result was that the ferrous oxide had split Into two phases, both of the same structure (rocksalt type), and, surprisingly, not of a decreased but an increased lattice parameter, compared with the oxide formed on non-coated iron under otherwise identical conditions. The accompanying photographs illustrate the effect. The measured dimensions were as follows:

The reason for this expansion is that the magneslum ions, instead of replacing ferrous ions in the wüstite lattice, occupy vacant latticepositions, until all 'holes' are flled. Thenceforward a normal con traction takes place. The theoretical saturation parameter, derived by extrapolating the known pure wüstite values to the equiatomic composition ( $\mathrm{FeO}$ ), and allowing for the difference in the ionic radi of $\mathrm{Fe}++$ and $\mathrm{Mg}++$, is $4 \cdot 328 \mathrm{~A}$., assuming all vacant sites in singlephase wüstite to be occupied. This would mean an expansion of the cube edge of $4 \cdot 328-4 \cdot 284=0.044 \mathrm{~A}$., compared with uncoated fron. The actual expansion, being divided between two phases of practically equal amounts, is $\frac{1}{2}[(4 \cdot 298-4 \cdot 284)+(4 \cdot 317-4 \cdot 284)]=0.023 \mathrm{~A}$. that is, in the present case occupation has proceeded to approximately half the saturation value.

Thus it can be sald that the defect lattice of wustite has been 'impregnated' by magnesium lons. Since vacant metallic lattice site are liable to aid outward diffusion of anions (FeO in scales affords poor protection against further oxidation), the question arises whethe by such impregnation the corrosion resistance can bo improved. According to Wagner ${ }^{1}$, the defleiency of metal ions in oxide structures (for example, $\mathrm{Cu}_{2} \mathrm{O}, \mathrm{FeO}$ ) increases the electrical conductivity and also explains the greater oxidizability of iron compared with other metals (compare, for example, Pfeil's work ${ }^{2}$ ). This is reflected by the considerable decrease in $\mathrm{Fe}: 0$ ratio of the ferrous oxide phase, below the value of the stoichlometrical composition, from the iron/scale to the scale/oxygen interface, revorted, for example, by the writer.

Quantitative hot oxidation tests in pure iron, with and withont magnesium oxide covering carried out to detect any protective effect due to the impregnation, have so far shown no improvement of cor- 